\title{
Implementación de indicadores de gestión por procesos para empresas de desarrollo de software
}

\section{Implementation of Process Management indicators for Software Development Companies}

\author{
Galo Hurtadoํㅗㄹ Max Zuñiga ${ }^{2}$, Santiago Durazno 3 \\ 1. Docente Investigador, Instituto Superior Tecnológico Particular Sudamericano, Cuenca- Ecuador. \\ Email: gphurtado@sudamericano.edu.ec ORCID: http://orcid.org/0000-0002-7190-140X \\ 2. Docente Investigador, Instituto Superior Tecnológico Particular Sudamericano, Cuenca- Ecuador. \\ Email: mzuniga@sudamericano.edu.ec ORCID: https://orcid.org/0000-0002-3720-562X \\ 3. Docente Investigador, Instituto Superior Tecnológico Particular Sudamericano, Cuenca- Ecuador. \\ Email: tsdurazno@sudamericano.edu.ec ORCID: https://orcid.org/0000-0003-3047-5296
}

Para Citar: Hurtado, G., Zuñiga, M., \& Durazno, S. (2020). Implementación de indicadores de gestión por procesos para empresas de desarrollo de software. Revista Publicando, 7(25), 170-179. Recuperado a partir de https://revistapublicando.org/revista/index.php/crv/article/view/2101

Resumen: El objetivo de la investigación se enfoca en el desarrollo de software al momento de emprender, que tenga una herramienta o pueda proveerse de las herramientas teóricas y metodológicas necesarias para la concepción e implantación de Sistemas de Gestión, que van desde la planeación estratégica y la gestión por procesos, alcanzando con esto los resultados esperados es decir cumpliendo los objetivos estratégicos de la empresa, permitiendo de esta manera resolver unas de las mayores interrogantes al momento de emprender que son ¿cómo se puede afrontar a la competencia? ¿Cuál es el valor agregado que les ofrece a los clientes? Para poder responder a las interrogantes fue necesario realizar un análisis de la realidad de las empresas desarrolladoras de software en diferentes sectores y con distinta población, para el caso de estudio se tomaron empresas de la ciudad de Cuenca. De igual manera, la investigación permite la alineación de los objetivos estratégicos con los procesos de la empresa, estableciendo una metodología que posibilita al desarrollador implementar una serie de pasos que van desde la toma de requerimientos hasta la entrega final del aplicativo al cliente; también facilita el trabajo con el uso adecuado de herramientas necesarias que permiten optimizar el tiempo y los costos.

Palabras clave: Gestión, procesos, empresas, desarrollo, software.

\begin{abstract}
The objective of the research is focused on the software developer at the time of undertaking, who has a tool or can provide the theoretical and methodological tools necessary for the design and implementation of Management Systems, ranging from strategic planning and process management, thus achieving the expected results that is to say, fulfilling the strategic objectives of the company, thus allowing to solve one of the biggest questions at the time of undertaking that are how to face the competition? What is the added value they offer to customers? To answer these questions, an analysis of the reality of software development companies in different sectors and with different populations must be carried out.

In the same way the article will allow to establish the alignment of the strategic objectives with the processes of the company, establishing a methodology that allows the developer to establish a series of steps to follow that go from the taking of requirements to the final delivery of the application to the client, this way also will be able to facilitate the work with the suitable use of necessary tools that allow to optimize the time and the costs.
\end{abstract}

Keywords: Management, processes, companies, development, software. 


\section{INTRODUCCIÓN}

$\mathrm{S}$

e plantea un instructivo que permite a las empresas micro, pequeñas, medianas o grandes desarrollar modelos de gestión, desde los más básicos hasta modelos de gestión macros, es decir se puede partir desde una base y a medida que la empresa crece, se podrían ir implementando los nuevos módulos de gestión. Un estudio realizado menciona que toda empresa necesariamente debe contar con un modelo de gestión (Canela, 2004).

En la actualidad el emprendimiento se ha vuelto una necesidad debido a muchos factores que influyen en el sector económico, uno de ellos es la falta de empleo (Kirberg, 2014), lo que conlleva a ciertas personas o grupo de personas a iniciar su propio negocio o pequeñas empresas, que en muchas ocasiones al momento de emprender cometen una serie de errores que se convierten en factores para que no progrese el emprendimiento, en el caso de estudio en particular se realiza un análisis de las empresas de desarrollo de software de la ciudad de cuenca y se trabaja sobre los modelos de gestión de procesos logrando de esta manera determinar cuál es el grado de importancia de la implantación de los modelos para el desarrollo de las empresas.

En el caso de estudio se toman como ejemplo tres empresas para proponer la implementación de modelos de gestión por procesos, identificando los diferentes problemas o soluciones que aportaría un buen modelo de gestión de procesos, cabe recalcar que el modelo de gestión dependerá de factores netamente de la empresa, considerando que cada una tiene diferentes procesos, el trabajo servirá de aporte para las pequeñas, medianas o grandes empresas que no cuentan con un diagrama de gestión por procesos, más bien lo llevan de una forma tradicional es decir lo hace en ese momento dependiendo de la necesidad del cliente y según Delfín y Acosta (2016), menciona que en muchos de los casos se ha perdido una gran cantidad de dinero por una gestión al momento de negociación con los clientes. Uno de los objetivos de la investigación es que el emprendedor pueda determinar con claridad cuál es el mejor camino o herramienta a seguir, para lo cual, mediante la revisión bibliográfica y diferentes revistas de impacto tecnológico se determinó que existen dos tipos de metodologías a seguir, la tradicional y la ágil.
Se debe tener en consideración que al igual que cualquier otro producto, el desarrollo de software tiene que pasar por ciertas etapas o procesos, los mismos tienen que cumplir con parámetros que garanticen la calidad de producto final, el que se entregará al usuario.

Para cumplir con los estándares de calidad es recomendable que se cumpla con ciertas normas de estandarización como la ISO/IEC 9126 de calidad de software (Mera, 2016), la que garantizará que sea un producto útil y agregue el valor de ser medible por quienes los producen y quienes los utilicen, de esta manera se cumplen los siguientes requerimientos planteados en el sistema de gestión de calidad (S.G.C):

- Entradas y salidas: teniendo en consideración cuál va a ser la transformación de valor.

- Secuencia e interacción: establecer los parámetros del proveedor y del cliente final.

- Criterios y métodos: Asegurar una operación eficaz y bajo control.

- Recursos necesarios: identificar bien los recursos a ser utilizados en la producción.

- Asignar responsables: quien responde por las actividades realizadas.

- Riesgos y oportunidades: disminuir los riesgos y aprovechar las oportunidades.

- Evaluar: implementar cambios si es necesario para lograr los objetivos planteados con indicadores operacionales o de gestión.

- Mejorar: Salir de la zona de confort e innovar adaptarse al cambio constante y las necesidades del entorno.

Las recomendaciones que realizan los autores Cervantes et al. (2016), es que un sistema de software puede establecerse como una transformación hacia una solución técnica de determinada problemática, para lo cual se pueden establecer una serie de actividades, entre las principales se plantea: 
Figura 1. Etapas del desarrollo de un sistema

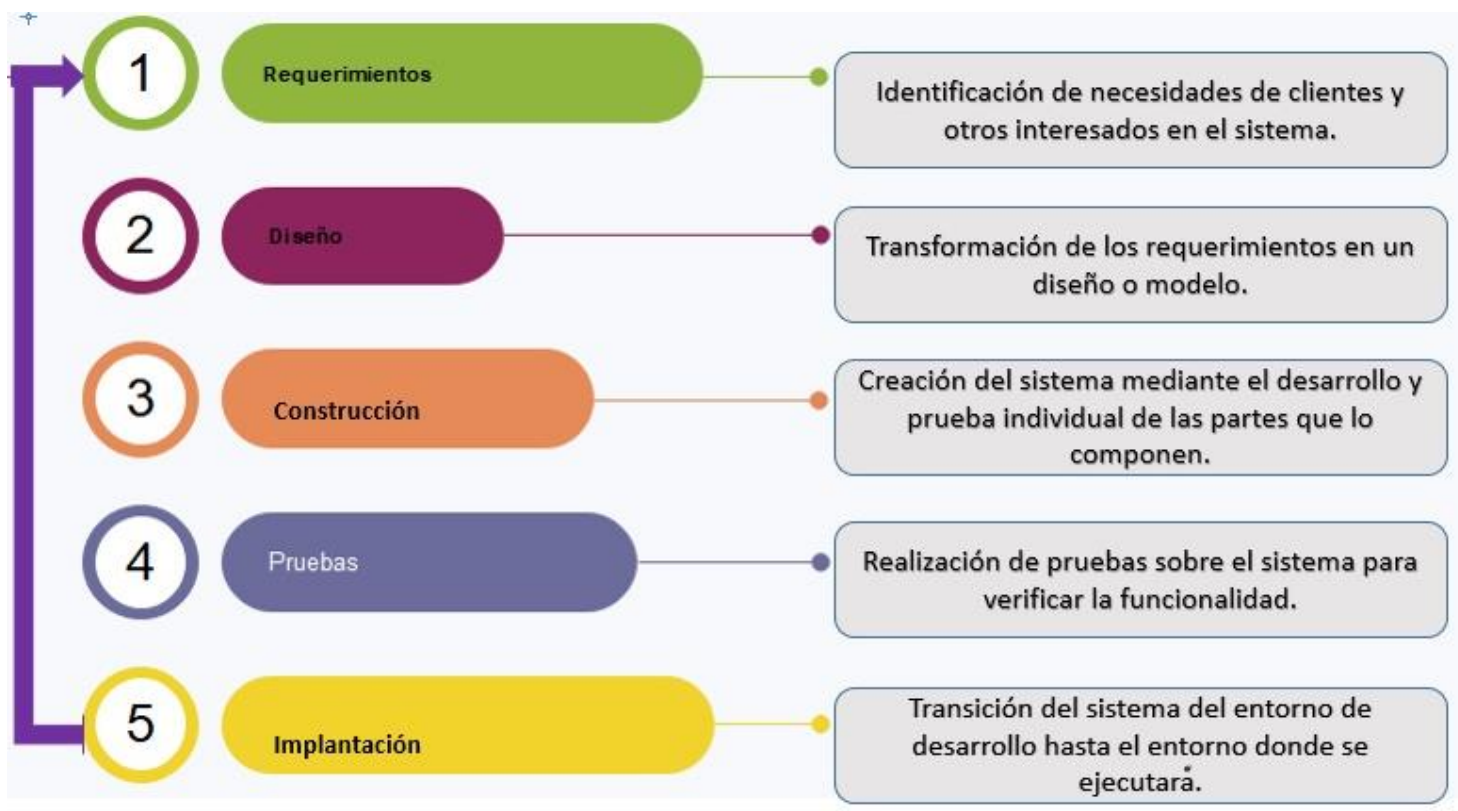

Fuente: (Hurtado Crespo, 2017).

Los procesos sirven para generar un valor agregado (Morillo, 2005), es el documento u objeto en el cual reposa la información de la empresa, de cada uno de los diferentes departamentos, se debe tener en consideración también que cada proceso tiene que tener un enfoque como se detalla a continuación:

- Definir las actividades claras y concisas que permitan cumplir los objetivos planeados.

- Establecer responsables por procesos para las actividades clave.

- Relacionar a los responsables de los diferentes departamentos con la finalidad de generar soluciones en bien de la empresa.

- Evaluar las incidencias de las actividades para la satisfacción del cliente.

Para establecer de manera clara y concisa todos estos pasos, se recomienda realizar un diagrama de flujo, que es una forma de traducir los requerimientos de los usuarios a un lenguaje más entendible por los desarrolladores de software, así lo menciona Yanes y Gaitan (2005), también afirman que se puede realizar la prueba de la necesidad que constituye el primer paso para implantar un sistema de gestión total por la eficiencia al momento de planificar un proyecto.

El objetivo de establecer un enfoque de procesos es el uso eficaz de los recursos, así como la reducción en tiempo y costo, se puede también centralizar las oportunidades de mejora. Cabe recalcar que las empresas se clasifican de acuerdo con ciertos parámetros así lo menciona:

Tabla 1

Clasificación de las empresas según el número de empleados

\begin{tabular}{ll}
\hline Tamaño & Número de Empleados \\
\hline Micro & $0-19$ \\
Pequeña & $20-49$ \\
Mediana & $50-99$ \\
Grande & Más de 100 \\
\hline
\end{tabular}

Fuente: (Nieto, V. et al.,2015). 
De esta manera se puede clasificar a las empresas según el número de empleados, entonces las empresas en las que se realizó el caso de estudio son una micro, una pequeña y una mediana que se describen a continuación.

\section{MATERIALES Y MÉTODOS}

Se presenta una investigación explicativa ya que se realiza un análisis entre diferentes tipos de empresas: micro, pequeñas, medianas y grandes; para la evaluación de resultados se utilizó una metodología cualitativa y se genera una guía o modelo de gestión que se pueda establecer al momento de emprender.

\section{INDICADORES DE GESTIÓN}

En la actualidad y gracias al avance de la tecnología se cuenta con varias herramientas para realizar diagramas de gestión, ya sean de paga o gratuitas las herramientas optimizan el uso de recursos y permiten al empresario determinar si se cumplen o no los objetivos estratégicos de la empresa, incluso saber cuál es el punto más bajo para establecer una mejora continua para lo cual Giraldo y Mauricio (2013) recomiendan establecer los siguientes indicadores de gestión:

\section{Objetivo:}

2. Servirá para determinar el para qué se quiere auditar o revisar cierto parámetro, es decir permitirá establecer acciones preventivas o correctivas en una misma dirección que será la de mejorar la calidad del producto final (Arita et al., 2005). Medida: Se realizarán operaciones matemáticas que permitan conocer cuál es el estado del parámetro que se está analizando.

\section{Niveles de referencia:}

Permite establecer una comparación con la cual contrastar el valor de un indicador; se recomiendan algunos niveles a ser analizados, entre ellos se encuentran los siguientes:

\section{Tabla 2}

Clasificación de los niveles de indicadores

\begin{tabular}{ll}
\hline Nivel Histórico & Nivel de competencia \\
\hline Nivel Estándar & $\begin{array}{l}\text { Nivel consideración } \\
\text { política }\end{array}$ \\
Nivel Teórico & Nivel conceso \\
Requerimientos de los usuarios & Nivel Responsabilidad
\end{tabular}

Fuente: (Hurtado, 2017).

Los indicadores permitirán tomar las acciones oportunas en cada una de las áreas de la empresa y aplicar las diferentes medidas establecidas en los siguientes niveles de referencia:

Figura 2. Niveles de referencia.

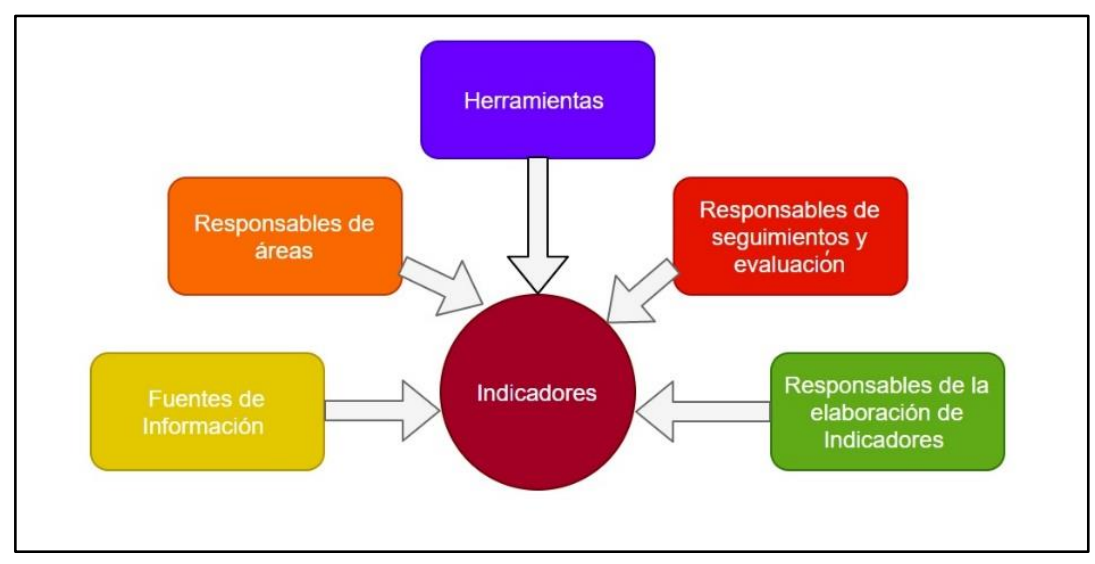

Fuente: (Hurtado, 2017) 
4. Instrumentos:

Se establece la forma en la que se aplicarán las diferentes métricas, para de acuerdo con los resultados generar información que permita a los responsables tomar decisiones.

\section{Periodicidad:}

Se sugiere establecer tiempos específicos para llevar a cabo las acciones antes mencionadas.

\section{Sistemas de Información:}

Se debe realizar un análisis correcto de la información que se obtiene para realizar una correcta toma de decisiones.

\section{Características de los indicadores}

Según Salgueiro (2001) recomienda algunos puntos relevantes para establecer un buen indicador entre los cuales se plantean:

1. Disponibilidad: los datos que se obtienen para la elaboración de un indicador deben ser de fácil acceso y estar disponibles todo el tiempo para el análisis o evaluación de este.

2. Simplicidad: debe ser lo más claro y fácil de interpretar.

3. Validez: un buen indicador debe generar como resultado lo que realmente se pidió es decir medir lo que uno desea.

4. Especificidad: si un indicador no mide realmente lo que se desea medir, su valor es limitado, por lo tanto, no servirá para la toma de decisiones.

5. Confiabilidad: las fuentes de información deben cumplir con características que garanticen la fidelidad de los datos.

6. Alcance: servirá para determinar cuál es el límite de dicho indicador.

Los indicadores son la parte más importante de la empresa, si se toman en consideración todos los parámetros antes mencionados se podrán tomar las decisiones acertadas en cada uno de los casos que se puedan presentar, optimizando y garantizando la calidad del producto o servicio que oferte la empresa (Pérez \& Mesanat, 2006). De igual manera para poder evaluar los indicadores se propone generar una matriz de mejora continua que se plantea a continuación:

Figura 3. Matriz de mejora continua.

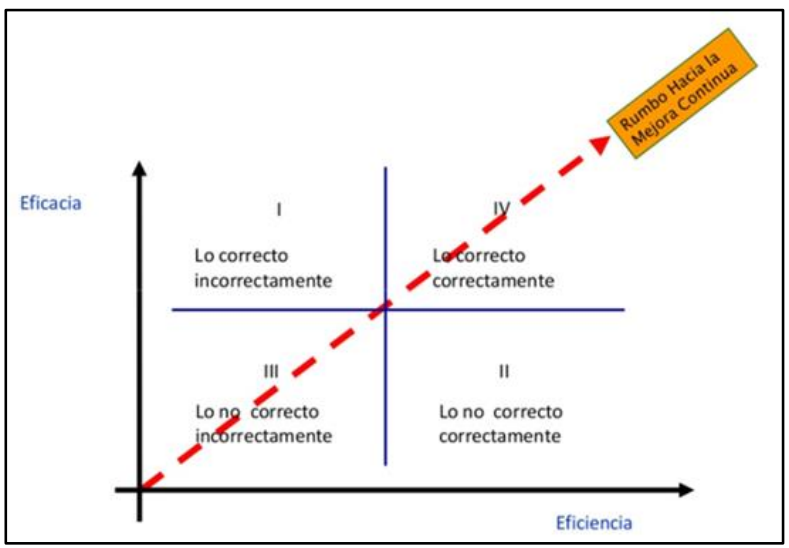

Fuente: (Gómez-Rivero \& Marin, 2018).

De esta manera se puede medir la eficacia, eficiencia y productividad, para poder obtener los resultados se tienen que establecer razones que relacionen la oportunidad, el costo y demás aspectos de calidad representándolos de la siguiente manera:

$$
\text { Eficacia }=\frac{\text { Resultados Obtenidos }}{\text { Resultados Requeridos }}
$$

También se aplicará una fórmula que permita calcular la eficiencia y optimizar recursos, únicamente se tiene que aplicar la siguiente relación:

$$
\text { Eficiencia }=\frac{\text { Resultados Utilizados }}{\text { Recursos Presupuestados }}
$$

Se puede aplicar la fórmula para obtener resultados de eficacia financiera, de mano de obra, tiempos, entre otros valores que se puedan relacionar. Para poder medir la productividad y medir cuál es el uso que se le está dando a los recursos de la empresa se tiene que establecer la siguiente formula: 


$$
\text { Productividad }=\frac{\text { Resultados Obtenidos }}{\text { Recursos Utilizados }}
$$

Aplicando las fórmulas antes mencionadas las empresas con independencia del tamaño podrán obtener resultados que permitan la toma de decisiones de una manera acertada, pudiendo establecer acciones preventivas o correctivas que mejoren la productividad.

\section{RESULTADOS}

Para la obtención de resultados se aplicó la investigación de campo que se llevó a cabo con cada uno de los gerentes de las empresas estudiadas de la ciudad de Cuenca, que a su vez tienen como actividad comercial el Desarrollo de Software, se aplicó la investigación en el producto desarrollo de páginas web, las empresas por su tamaño se han clasificado en micro, pequeñas, medianas y grandes, cabe recalcar que si bien las empresas tienen sus años de trayectoria, en ninguno de los casos contaban con un modelo de gestión diseñado más bien se realizaban todos los procesos de manera empírica, a continuación, se presentan las empresas en las que se implementó un modelo de gestión:

\section{SONEXT}

Considerada por el número de empleados como una microempresa, se encarga del desarrollo de páginas y aplicaciones web, por medio de los datos recolectados, se plantea el siguiente modelo de gestión que permite realizar la entrega de los trabajos en los tiempos establecidos con los clientes.

Figura 1. Diagrama de gestión propuesto para la empresa.

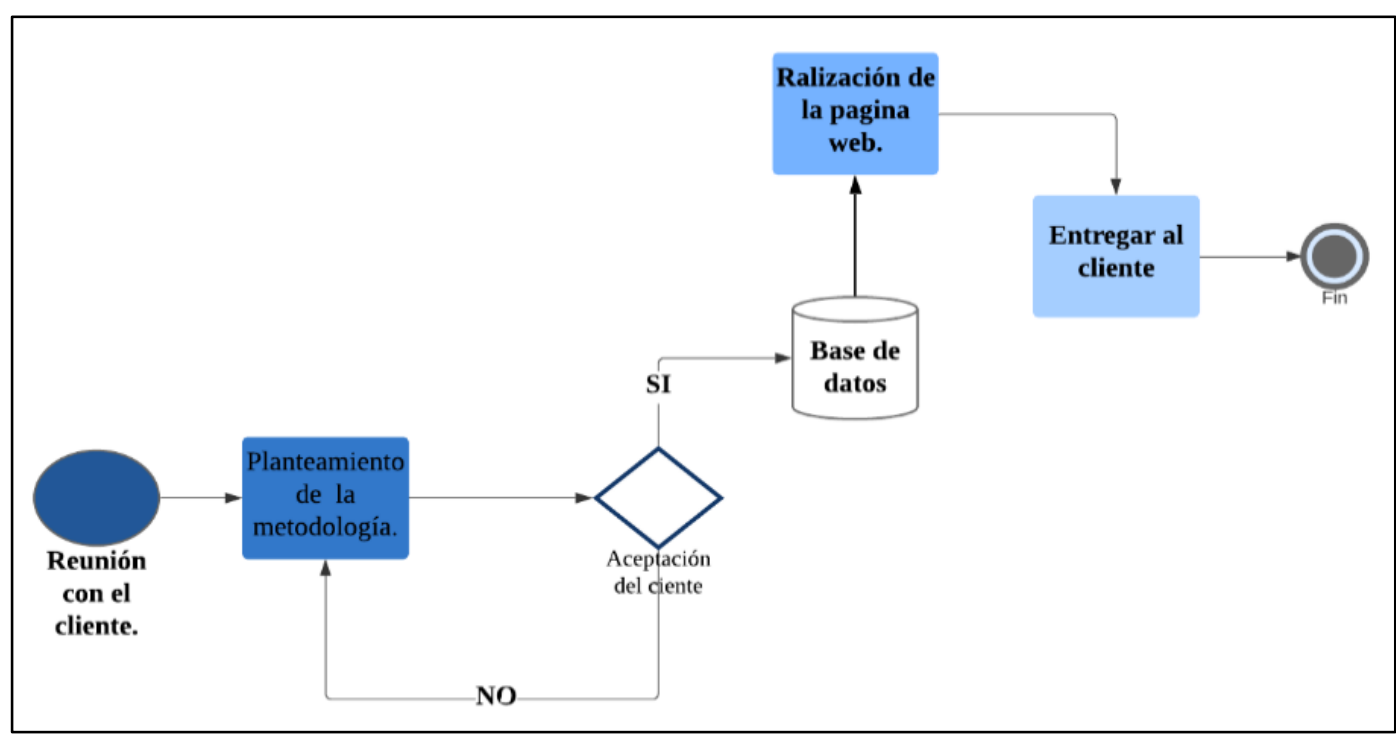

Fuente: (Hurtado, 2017)

\section{MYCODEDMIND}

La empresa por el número de empleados se considera pequeña, la cual brinda servicios integrales de comunicaciones corporativas y publicitarias en medios digitales, se presentó una propuesta de gestión de procesos para el desarrollo de páginas web. 
Figura 2 Diagrama de gestión propuesto para la empresa.

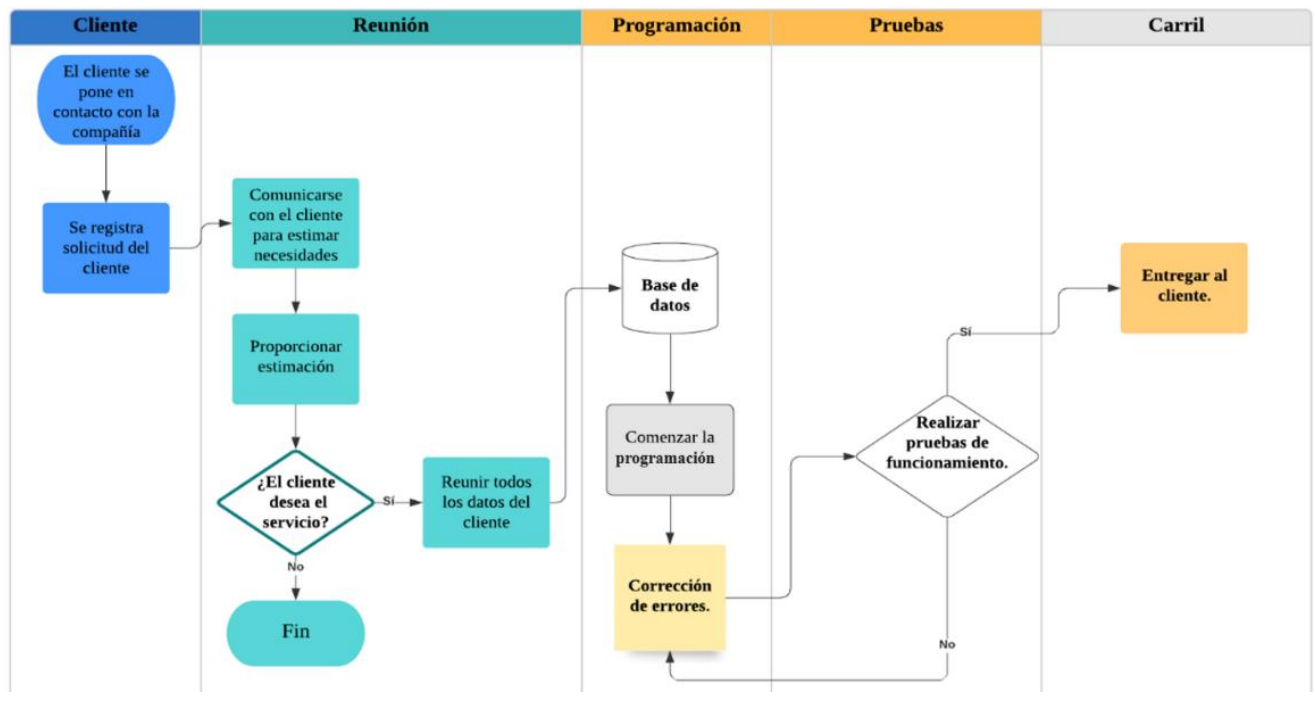

Fuente: (Hurtado, 2017)

\section{RIVERMIND}

La empresa "RIVERMINDS", por el número de empleados se considera mediana, se dedica a la implementación de soluciones informáticas, se le realizó una entrevista para descubrir los pasos que sigue al momento de desarrollar una página web, una vez recopilada la información se plantea el siguiente diagrama de gestión:

Figura 3 Diagrama de gestión propuesto para la empresa

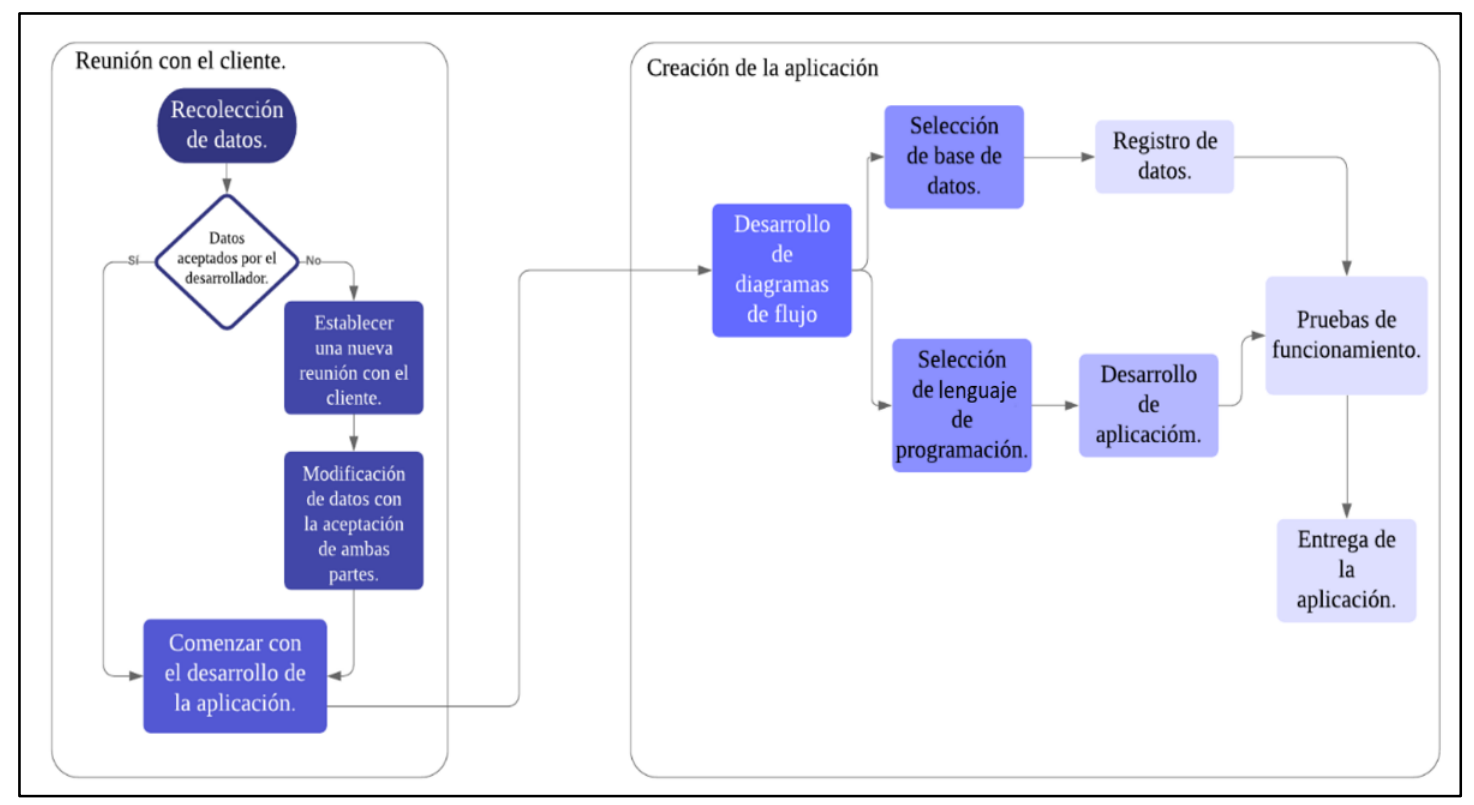

Fuente: (Hurtado, 2017)

Hurtado, G., Zuñiga, M., \& Durazno, S. (2020). Implementación de indicadores de gestión por procesos para empresas de desarrollo de 
Se realizó mediante la fórmula un análisis de producción de los tres últimos meses en cada una de las empresas obteniendo los siguientes resultados:

Tabla 3. Análisis de producción.

\begin{tabular}{|c|c|c|c|}
\hline & Eficacia & Eficiencia & Productividad \\
\hline \multirow[t]{2}{*}{ Fórmula } & \multirow{2}{*}{$\frac{\text { Pag. Producidas }}{\text { Paginas Programadas }}$} & \multirow{2}{*}{$\begin{array}{l}E f i= \\
\frac{\text { Dinero Utilizado }}{\text { Dinero Presupuest. }} C / \text { pag. }\end{array}$} & \multirow{2}{*}{$\begin{array}{l}\text { Prod }=\frac{\text { Personal Labora }}{\text { Pag.Producidas }} \\
\text { c/pag. }\end{array}$} \\
\hline & & & \\
\hline \multirow[t]{3}{*}{ SONEXT } & & $E f i=\frac{400}{600}=0,6$ & \multirow{3}{*}{$\begin{array}{l}\text { Prod }=\frac{4}{8}=0,5 \\
50 \% \text { de productividad }\end{array}$} \\
\hline & Eficacia $=\frac{12}{}$ & & \\
\hline & $=0,8 \quad 80 \%$ & Generando una utilidad del 40\% & \\
\hline \multirow[t]{3}{*}{ MYCODEDMIND } & & $E f i=\frac{480}{600}=0,8$ & \multirow{3}{*}{$\begin{array}{l}\text { Prod }=\frac{6}{15}=0,4 \\
40 \% \text { de productividad }\end{array}$} \\
\hline & Eficacia $=\frac{17}{21}$ & & \\
\hline & $=0,8181 \%^{21}$ & Generando una utilidad del 20\% & \\
\hline \multirow[t]{3}{*}{ RIVERMIND } & & $E f i=\frac{500}{600}=0,83$ & \multirow{3}{*}{$\begin{array}{l}\text { Prod }=\frac{9}{27}=0,33 \\
33 \% \text { de productividad }\end{array}$} \\
\hline & Eficacia $=\frac{27}{2}$ & & \\
\hline & $=0,990 \%$ & Generando una utilidad del 17\% & \\
\hline
\end{tabular}

Fuente: (Hurtado, 2017)

A continuación, se presenta un análisis del rendimiento de las empresas en los últimos tres meses:

Figura 4 Análisis de resultados

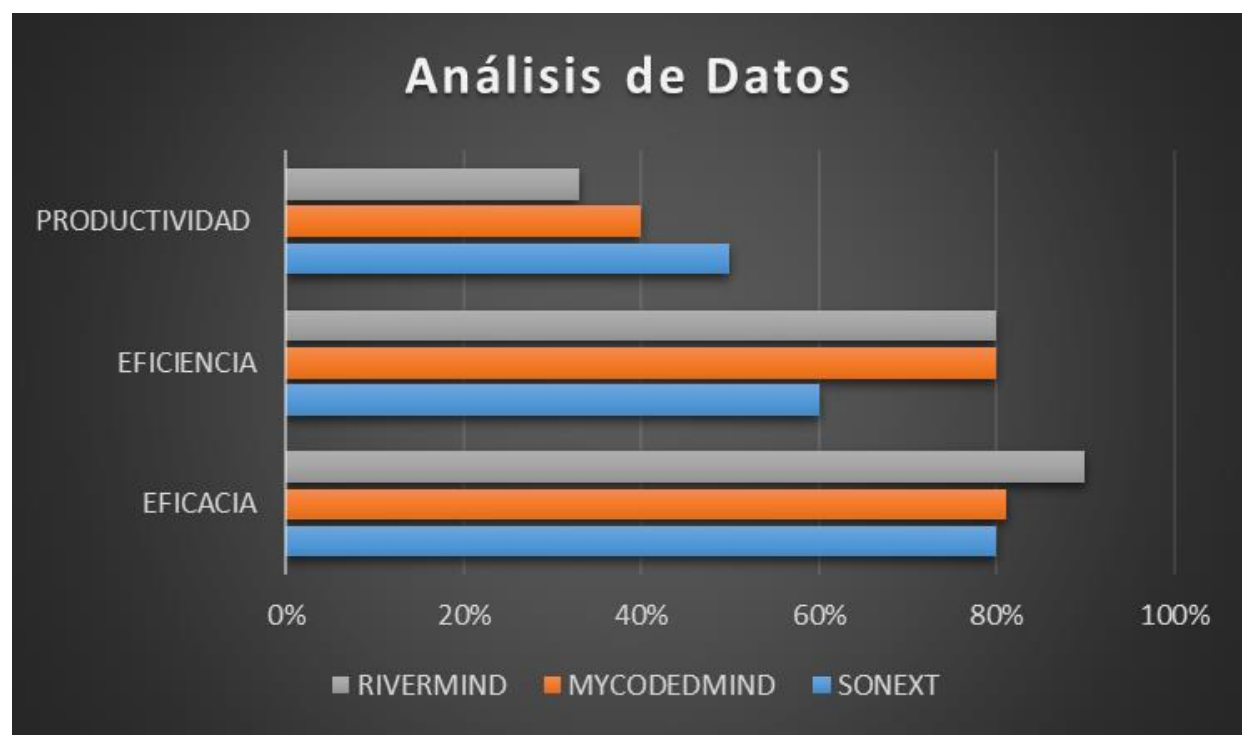

Fuente: (Hurtado, 2017). 
Con la aplicación de las fórmulas se obtuvieron los datos que se observan en la gráfica anterior, se puede también determinar que la productividad dependerá del número de páginas web que se produzcan al mes y del número de personal responsable de dichas páginas.

\section{CONCLUSIONES}

Esta publicación expone los resultados parciales del proyecto de investigación titulado: Realidad de la industria del software, el cual se desarrolla mediante el financiamiento del Instituto Tecnológico Superior Sudamericano. La implementación de la mayoría de las fases comprendidas en la investigación permite llegar a las siguientes conclusiones:

1. Se tienen que plantear los indicadores de una forma clara y concisa, ya que los mismos servirán para la toma de decisiones y aplicación de acciones preventivas o correctivas así también se tienen que concretar los objetivos de los indicadores definiendo el alcance y que a su vez sean medibles, generar indicadores que se apliquen periódicamente será indispensable para el análisis de resultados en cuanto a eficacia, eficiencia y productividad.

2. Las fórmulas que se plantean para el análisis de resultados se pueden plantear para micro, pequeñas, medianas y grandes empresas, que a su vez permitirán establecer parámetros para el análisis como la eficacia, eficiencia y productividad, estas métricas de control se pueden aplicar en cada uno de los parámetros de la empresa que se definieron en los diagramas de gestión.

3. Los diagramas de gestión son una herramienta indispensable al momento de evaluar el rendimiento de la empresa, permiten reconocer el punto específico que necesita ser mejorado o modificado, de esta

manera se demuestra la importancia de implementar un modelo de gestión por procesos en las empresas independiente del tamaño de esta. 


\section{REFERENCIAS BIBLIOGRÁFICAS}

Arita, B. Y., Romano, S., García, N., \& del Refugio Félix, M. (2005). Indicadores objetivos y subjetivos de la calidad de vida. Enseñanza e Investigación En Psicología, 10(1), 93-102.

\section{Google Scholar}

Canela López, J. R. (2004). La gestión por Calidad Total en la empresa moderna. México: ALFA OMEGA GRUPO EDITOR SA de CV.

\section{Google Scholar}

Delfín Pozos, F. L., \& Acosta Márquez, M. P. (2016). Importancia y análisis del desarrollo empresarial. Revista científica Pensamiento y gestión, (40).

\section{Google Scholar}

Giraldo, R., \& Mauricio, R. (2013). Seguimiento, Medición, Análisis y Mejora en los sistemas de Gestión, enfoque bajo indicadores de Gestión y Balanced Scorecard. ICONTEC.

\section{Google Scholar}

Gómez-Rivero, J., \& Marin, J. L. (2018). Modelo de evaluación de la capacidad de gestión de innovación en los institutos educación superior en el marco de la sociedad del conocimiento.

\section{Google Scholar}

Hurtado Crespo, G. P. (2017). Estudio comparativo entre servidores Mikrotik y Cisco bajo el estándar de seguridad 802.1 x para servicios de red en la empresa Guano. Net. Escuela Superior Politécnica de Chimborazo.

Google Scholar

Kirberg, A. S. (2014). Emprendimiento exitoso. Ecoe Ediciones.

Google Scholar
Mera Paz, J. A. (2016). Análisis del proceso de pruebas de calidad de software.

\section{Google Scholar}

Morillo, M. C. (2005). Análisis de la cadena de valor industrial y de la cadena de valor agregado para las pequeñas y medianas industrias. Actualidad Contable FACES, 8(10), 53-70.

\section{Google Scholar}

Nieto, V., Timoté, J. A., Sánchez, A. F., \& Villarreal, S. (2015). La clasificación por tamaño empresarial en Colombia: Historia y limitaciones para una propuesta. Archivos de Economía, 434.

\section{Google Scholar}

Pérez, A. S., \& Mesanat, G. G. (2006). ¿ Qué indica un indicador?: análisis comparativo en los destinos turísticos. Journal of Tourism Analysis= Revista de Análisis Turístico, (2), 69-85.

\section{Google Scholar}

Salgueiro, A. (2001). Indicadores de gestión y cuadro de mando. Ediciones Díaz de Santos.

\section{Google Scholar}

Yanes, J. P. M., \& Gaitan, O. G. (2005). Herramientas para la gestión energética empresarial. Scientia et Technica, 3(29). 